\title{
Communication Signal Modulation Mechanism Based on Artificial Feature Engineering Deep Neural Network Modulation Identifier
}

\author{
Fei Lu $\mathbb{D}^{1},{ }^{1}$ Zhenjiang Shi $\mathbb{D}^{1},{ }^{1}$ and Rijian Su $\mathbb{C}^{2}$ \\ ${ }^{1}$ Department of Engineering Technology, Open University of Guangdong (Guangdong Polytechnic Institute), Guangzhou, \\ Guangdong 510091, China \\ ${ }^{2}$ School of Computer and Communication Engineering, Zhengzhou University of Light Industry, Zhengzhou, Henan 450000, China
}

Correspondence should be addressed to Rijian Su; srj@zzuli.edu.cn

Received 29 March 2021; Revised 24 May 2021; Accepted 31 May 2021; Published 18 June 2021

Academic Editor: Wei Wang

Copyright (C) 2021 Fei Lu et al. This is an open access article distributed under the Creative Commons Attribution License, which permits unrestricted use, distribution, and reproduction in any medium, provided the original work is properly cited.

\begin{abstract}
Based on the characteristics of time domain and frequency domain recognition theory, a recognition scheme is designed to complete the modulation identification of communication signals including 16 analog and digital modulations, involving 10 different eigenvalues in total. In the in-class recognition of FSK signal, feature extraction in frequency domain is carried out, and a statistical algorithm of spectral peak number is proposed. This paper presents a method to calculate the rotation degree of constellation image. By calculating the rotation degree and modifying the clustering radius, the recognition rate of QAM signal is improved significantly. Another commonly used method for calculating the rotation of constellations is based on Radon transform. Compared with the proposed algorithm, the proposed algorithm has lower computational complexity and higher accuracy under certain SNR conditions. In the modulation discriminator of the deep neural network, the spectral features and cumulative features are extracted as inputs, the modified linear elements are used as neuron activation functions, and the crossentropy is used as loss functions. In the modulation recognitor of deep neural network, deep neural network and cyclic neural network are constructed for modulation recognition of communication signals. The neural network automatic modulation recognizer is implemented on CPU and GPU, which verifies the recognition accuracy of communication signal modulation recognizer based on neural network. The experimental results show that the communication signal modulation recognizer based on artificial neural network has good classification accuracy in both the training set and the test set.
\end{abstract}

\section{Introduction}

Modulation recognition of communication signals has a wide range of application requirements in modern wireless communications [1]. In noncooperative communication, since the receiver does not know the communication parameters of the sender, especially the modulation mode, it cannot effectively perform subsequent operations such as demodulation [2]. Communication signal modulation recognition is widely used in noncooperative communication scenarios such as electronic countermeasures, communication reconnaissance, signal recognition, and electronic supervision. (2) In the cognitive radio system, the communication receiver hopes to realize a universal receiver to accurately receive the information sent by the sender
[3]. In the design of universal receiver, signal modulation identification is a key technology. Only when the modulation mode of the signal is accurately identified can the carrier frequency bandwidth and other information of the signal be estimated more accurately, so as to facilitate the subsequent demodulation and decoding of the signal. Therefore, it is of great significance to study the modulation recognition of communication signals. In today's increasingly complex wireless communication environment, the electromagnetic signal space is more and more complex, the transmission of information is more and more large, and the signal changes more and more quickly. Therefore, the research on automatic and high-speed modulation pattern recognition of multiclass communication modulation signals has a very high application value. 
The method of wavelet transform [4] is proposed to extract three different features for the recognition of MPSK and MFSK. A simple method based on high-order cumulants [5] is proposed for the classification of digital modulation schemes. It is proposed that ten different types of digital modulation can be identified by using the multilayer perceptron method [6] in combination with the spectrum and statistical accumulative characteristic sets of signals. In recent years, the modulation recognition algorithms are becoming ever more diverse. Random forests, decision trees, support vector machines, and other models are widely used [7, 8]. SVM occupies a dominant position in the field of machine learning due to its complete theoretical basis and good practical effects. Modulation recognizers based on the SVM model are emerging endlessly $[9,10]$. The essence is to slightly improve the SVM model based on the extracted communication signal feature vector, such as improving the multiclassification strategy of SVM, improving the kernel function of SVM, and improving the penalty term of SVM $[11,12]$. According to the different priori information obtained from the signal, the classical signal detection algorithm can be mainly divided into three categories: the signal detection algorithm based on matched filtering, the signal detection algorithm based on feature, and the signal detection algorithm based on energy detection. Matched filtering [13] algorithm is the best signal detection method in theory, but because it needs to obtain the priori information of the target signal in a comprehensive way, the application scene is strictly required in the actual application process. On the other hand, as the bandwidth of the target spectrum increases, when there are multiple signal forms in the detected frequency band, it is necessary to design a matched filter for each signal and compare it one by one, which is of great computational complexity. The feature-based signal detection algorithm requires less signal prior information and can obtain better detection performance under certain prior conditions. The method based on correlation detection is the most common one among feature detection algorithms. The main idea is to design correlation rules by using the inherent correlation characteristics of signals [14]. However, the performance of correlation detection depends heavily on the statistical characteristics of the signal to be detected, and the detection performance varies greatly with the difference of feature selection. The signal detection algorithm based on cyclic features [15] is also an algorithm with excellent performance and good antinoise performance. However, the cycle feature detection is not suitable for systems that require high real-time performance due to the large amount of calculation. In general, the feature-based signal detection method can obtain good detection accuracy in some cases, but the detection performance is also limited by prior information, and the performance is still limited under noncooperative conditions. Energy detection method $[16,17]$ takes the energy of the signal in the detection domain as the evaluation standard and can be divided into time domain detection and transformation domain detection according to different detection domains. Among them, the time domain detection method has poor antinoise ability and poor real-time performance, and its performance needs to be improved, and most of them are based on narrow-band detection tasks. In the transformation domain detection algorithm
$[18,19]$, the power spectrum method is widely used in practical engineering due to its advantages of clear and simple physical meaning, low computational complexity, and direct application in the field of multisignal detection. However, due to the influence of complex electromagnetic environment and partial impedance mismatch of receiver analog front end, the noise base of the obtained broadband signal may have a large fluctuation, and this fluctuation phenomenon will lead to a serious decline in detection performance. For this kind of situation, the power spectrum detection algorithm based on the correction of noise base has been widely studied [20, 21]. Its basic idea and technical route are as follows: estimate the noise base, use the estimated base to correct the power spectrum, and finally build the detection model based on the modified Gaussian white noise. The signal detection performance of this method depends heavily on the accuracy of the estimation of the noise base, and the robustness is poor in complex electromagnetic environment. Moreover, the detection algorithm based on power spectrum is only applicable to stationary signals. For nonstationary signals, the signal cannot be comprehensively described on the power spectrum because the spectrum structure may change with time $[22,23]$. Therefore, signal detection based on time-frequency domain method is also widely studied [24-29]. Due to the diversity of modulation methods, it is difficult to find a universal algorithm that can recognize all modulation methods so far. Different algorithms can be applied to different signal types. Therefore, in practice, it may be necessary to combine a variety of different algorithms to meet the expected requirements. The modulation recognition algorithms mainly fall into two categories: one is the likelihood ratio method based on hypothesis testing, which takes each modulation mode as a possible alternative hypothesis, and determines the modulation mode of the signal by constructing the likelihood function and obtaining the maximum likelihood; the second is the pattern recognition method based on feature extraction. The idea is to extract some feature information from the signal or extract some information and then carry out some operations to obtain some characteristic values, and through these information classifications, identify the modulation type. Past research communication signal modulation style intelligent recognition technology has a lot of research results; in some application scenarios, end-to-end algorithm is less than human characteristic modulation recognition algorithm based on artificial features, but the selection of model does not have definite theory support, and the degree of matching between input data and the model is much less than the reliability of the model which is often restricted to the adequacy of the data. The training of a large number of signal samples will inevitably lead to a large amount of time and resource consumption. The application of artificial features in the field of signal modulation recognition needs further research.

Based on deep neural network and artificial feature engineering, a communication signal modulation recognizer is constructed. In this paper, a concrete design scheme of neural network is proposed firstly, and then, a communication signal modulation recognitor is implemented on CPU and GPU, and the experimental performance is given. In addition, on the basis of studying the different features, a kind of quasirecognition signal set recognition scheme is designed. 


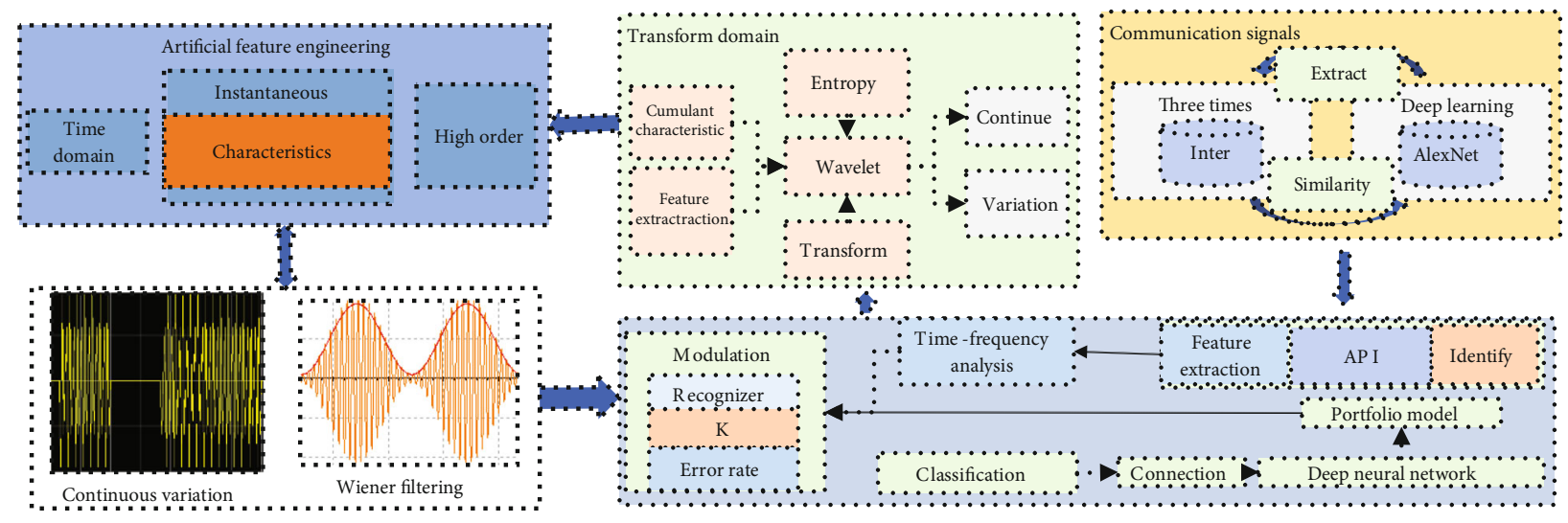

FIGURE 1: General framework of modulation recognition of artificial feature engineering deep neural network.

A statistical algorithm of spectral peak number for FS and $\mathrm{K}$ class recognition is proposed. In order to solve the problem of constellation rotation caused by phase mismatch of QAM signal during downconversion, an improvement was made. Finally, the whole recognition scheme is verified by simulation experiment.

\section{Modulation Mode and Artificial Feature Engineering Deep Neural Network Modulation Recognition for Communication Signals}

2.1. General Framework of Modulation Recognition for Artificial Feature Engineering Deep Neural Network. In order to realize the combination of artificial feature and deep neural network, the 20-dimensional artificial feature vector was constructed by designing and selecting the appropriate feature parameters, and the four-layer fully connected BP neural network was used to classify the artificial feature vector. Then, time-frequency analysis of the signals is carried out and appropriate preprocessing methods are selected. The modulation recognition algorithm based on deep learning is studied by using the AlexNet model and the InceptionResNet-V2 model, respectively, in order to realize the endto-end modulation recognition. Finally, based on the Indulge-ResNet-V2 model with better recognition effect, the existing model is improved by designing the combined model and the triple network model, and new signals outside the preset range are identified to expand the application range of the existing model, as shown in Figure 1.

In the construction of artificial feature vectors, extracting characteristic parameters with characterization ability from the received signals is the first step and also a key link in the realization of modulation recognition. In the recognition of MASK signal, MFSK signal, MPSK signal, and MQAM signal, three new transform domain features are designed, and on this basis, a 20-dimensional artificial feature vector is constructed, which contains six temporal instantaneous features, five highorder cumulant features, and nine transform domain features.

Different characteristic parameters can represent different signal modulation modes from different perspectives.
The difference of transient characteristics in time domain provides the intensity and stability of signal temporal waveform richness and instantaneous amplitude distribution, while high-order cumulative features can suppress the influence of Gaussian white noise and enhance the recognition effect at low SNR. The characteristics of the transform domain provide the information of discrete spectral line distribution, normalized spectral density, and instantaneous amplitude spectral density of the signal. The mind mapping of all feature parameters in the artificial feature vector is shown in Figure 2.

MASK, MQAM, MPSK, and MFSK signals vary in amplitude to different degrees. The envelope of MPSK signal is slightly undulating, while the envelope of MFSK signal is not undulating. These signals are different in terms of time domain waveform richness, intensity of instantaneous amplitude distribution and stability of amplitude information, etc. Six time domain instantaneous features are adopted, and the specific extraction algorithm of each characteristic parameter is as follows:

(1) Using the zero center of the signal to normalize the instantaneous amplitude, the characteristic parameters are obtained

Firstly, the zero center normalized instantaneous amplitude of the signal is calculated by using the instantaneous amplitude of the signal.

$$
\beta(n)=\frac{\beta(n)}{\sum_{n=1}^{N} \beta(n)}-1
$$

Then, a digital low-pass filter is used to smooth the filter by removing some burrs and amplitude mutations, and then, the parameters can be obtained by calculating the mean value and variance. If the filtered number sequence is $\beta(n)$, then the calculation expression of characteristic parameters is as follows:

$$
M_{\text {mean }}=\frac{1}{N \sum_{n} \beta(n)},
$$



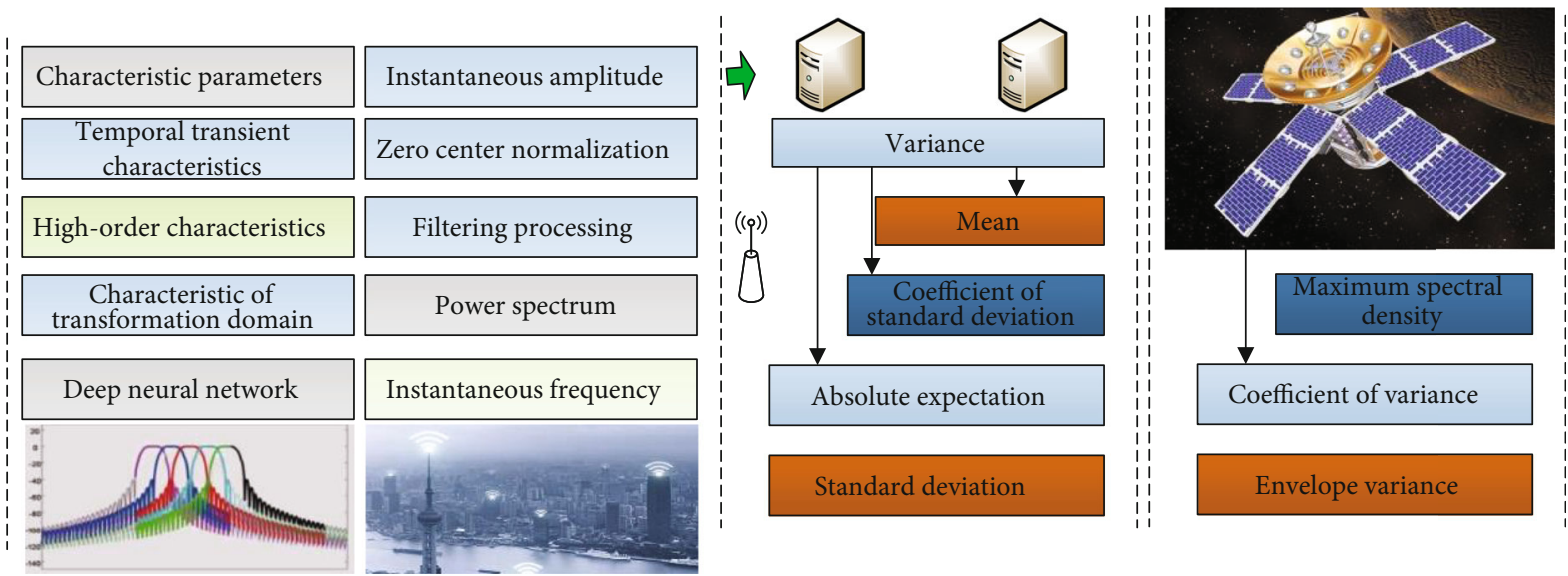

Figure 2: Modulation identification diagram of deep neural network for all feature parameters in artificial feature vectors.

$$
M_{2}=\frac{1}{M} \sum_{n}^{N}(\beta(m)-\overline{\beta(n)})^{2} .
$$

(2) Calculate the standard deviation coefficient and the expectation of absolute value of the two normalized instantaneous amplitudes of the zero center of the signal. The calculation expression is as follows

$$
M_{\beta}=E\left\|\beta_{N}\left(\beta_{n}(m)\right)\right\|
$$

(3) Calculate the standard deviation of the absolute value of the normalized instantaneous amplitude of the zero center of the differential signal, and obtain the characteristic parameters

$$
t a_{a c}=\sqrt{\frac{1}{M}\left\|\sum_{N} \beta^{2}(n)\right\|} .
$$

(4) The characteristic parameters can be obtained by calculating the variance coefficient of the envelope of the differential signal. Its calculation expression is as follows

$$
R=\frac{\beta^{2}}{m^{2}}
$$

The analysis of signal in time domain and transformation domain only describes the same existence from different perspectives. The transformation of perspectives often brings new information. Some signals that are difficult to see the features in time domain will be easier to see the features after being converted to transformation domain. Therefore, the characteristic parameters of the transformation domain also play an important role in modulation identification and can provide information on discrete spectral line distribution, normalized spectral density, instantaneous amplitude spectral density, and other aspects of the signal.

2.2. Research on Recognition of Modulation Mode of Communication Signal. Many studies use high-order cumulants or clustering algorithms to identify QAM signals in class. For QAM signal, using clustering recognition is the most direct method, but the cluster computing complexity is much higher than the level of higher-order cumulant, and higher-order cumulants also have some disadvantages; FS cumulant characteristic value $\mathrm{K}$ signal exactly makes impossible using higher-order cumulant characteristic value of FSK signal recognition, so the combination of the two methods is in this paper. Since high-order cumulants have lower operational complexity, it is preferred to use highorder cumulant features for in-class recognition of QAM signals. When the recognition cannot be completed by using high-order cumulant features, clustering is carried out. The theoretical values of high-order cumulants of the four QAM signals of 8QAM, 16QAM, 32QAM, and 64QAM are shown in Table 1:

The simulation shows that 16QAM and 64QAM cannot be distinguished effectively even with eighth-order cumulants. Therefore, after the signal set $\{16 \mathrm{QAM}, 64 \mathrm{QAM}\}$ is obtained by using $\mathrm{Ki}$ and $\mathrm{K}$ classifications, the clustering algorithm is used to separate the two. It is assumed that the clustering point is $M$, and the threshold value of the clustering point is $t(N 1)$, which is determined to be 64QAM when $M>t(M)$; otherwise, it is 16QAM.

In the case of phase asynchronization during mixing, as the constellation diagram rotates, the range of constellation points sampled at regular intervals is smaller than that in the case of phase synchronization, so the radius of clustering should be reduced accordingly. However, as the radius of subtraction clustering is set as a fixed value, the recognition rate of signals is reduced.

As can be seen in Figure 3, when the phase is out of sync, the constellation diagram rotates, and the clustering radius value should have been smaller. However, since its value 
TABLE 1: Theoretical value of higher-order cumulants of QAM signals.

\begin{tabular}{lcccc}
\hline Signal & 8QAM & 16QAM & 32QAM & 64QAM \\
\hline IC201 & 0 & 0 & 0 & 0 \\
IC211 & $0.668 E$ & $E$ & $E$ & $E$ \\
IC401 & $E$ & $0.68 E 2$ & $0.18 E 2$ & $0.61 E 2$ \\
IC411 & $E 2$ & 0 & 0 & 0.31 \\
\hline
\end{tabular}

has been fixed, for 64QAM signals, the clustering radius is relatively large, which leads to too few clustering points, thus leading to low recognition rate.

\subsection{Modulation Pattern Recognition of Communication} Signal Based on Modulation Recognizer of Deep Neural Network. With the improvement of computer processing speed and storage capacity, the design and implementation of CNN has gradually become a trend. Communication signal modulation pattern recognition method based on deep neural network is used to recognize modulation signal. Firstly, the received modulation signal is normalized and the time-frequency feature image is preprocessed to generate the training set and test set required for network training. Secondly, a classifier for communication signal modulation pattern recognition, namely, $\mathrm{CNN}$, is designed and built. The training set is input into $\mathrm{CNN}$ for training, and the CNN network model is obtained. Finally, the identified modulation signal is preprocessed to generate a test set in the dataset, and the training set is input into the CNN network model to identify the modulation mode of the communication signal. This method takes the time-frequency domain graph as input and the signal modulation mode as output. The specific algorithm flow chart is shown in Figure 4.

Time-frequency analysis represents nonstationary signals as two-dimensional functions are related to time and frequency, which can be analyzed and processed intuitively. It is a kind of important method for processing nonstationary signals. CWD is selected for time-frequency analysis of communication signal modulation mode, and then, the grayscale feature image generation algorithm is used to generate the training set and test set required by the network. Training set and testing set include 2ASK, 2FSK, 2PSK, five kinds of $\mathrm{AM}$ and FM modulation signal time-frequency characteristics of the image, SNR range for $[-\mathrm{SdB}, \mathrm{SdB}]$, interval of 1 $\mathrm{dB}$, each signal-to-noise ratio under each modulation signal sample for 200, including 60 for the test set and 140 as the training set, the training set including a total of 7700 samples, and a test set containing a total of 3300 samples.

After the design and construction of CNN's network structure is completed, some network super parameters, such as learning rate and weight attenuation coefficient, need to be set. The setting of super parameters in this network is shown in Table 2.

After setting the super parameters, the following network model training can be carried out. During training, 30\% of the training set is used as the validation set to verify the performance of the network. Through the training of the net-

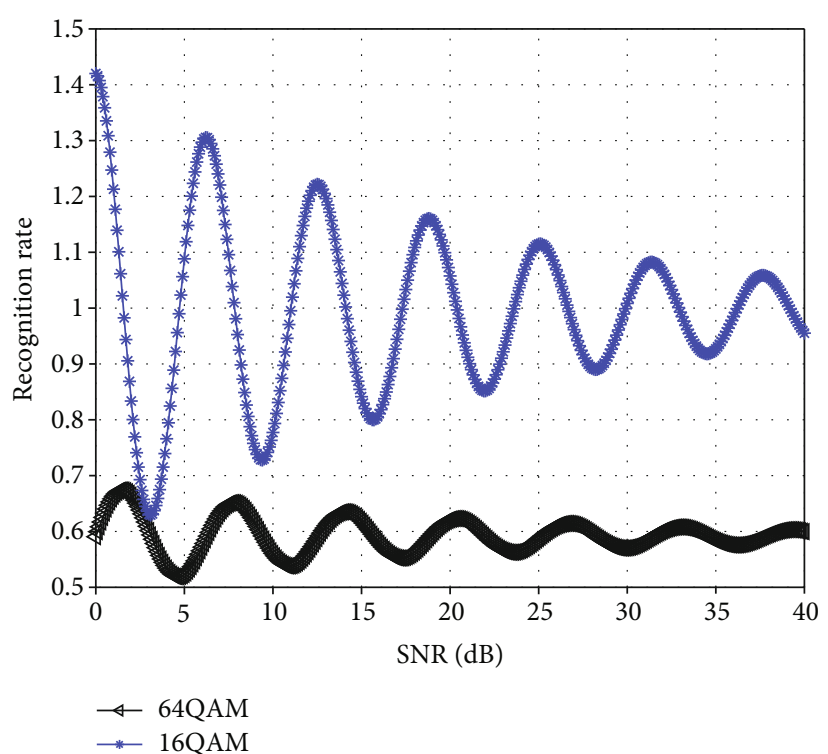

FiguRE 3: Recognition rate of subtraction clustering when the mixing phase difference is 45 degrees.

work, Figure 5 shows the loss value of the training set and the relationship between the accuracy and the number of iterations.

As can be seen from Figure 5, after 40 iterations of training, the accuracy of training set (ACC) is basically stable at about 0.99 , while the loss value of training set is also stable at about 0.15 . The accuracy of the verification set fluctuates around 0.85 , while the loss value of the verification set fluctuates greatly and shows an oscillating trend.

The first step is data preparation: in the deep neural network modulation identifier, the input is directly the original communication signal sampling data, and there is no need for feature extraction and preprocessing, but the appropriate dimension transformation must be carried out to adapt to the deep network input. Note that our three deep networks are input dimensions that are all (None, 1024, 1), which is a three-dimensional tensor: the first dimension None represents the number of samples and does not need to be specified. The second dimension, 1024, means the length of our communication signal is 1024 . Dimension 1 means there is only one channel. As for the label calibration of modulation mode, label unique thermal vectorization, and the division of training set and test set, it is completely consistent with the description in the implementation of modulation recognizer of deep neural network.

The second step is to build the model: based on the Keras and TensorFlow libraries, three kinds of deep neural networks are cooperatively built on the CPU and GPU computing platform according to the above network architecture description.

The third step is training model and test: with good training, dataset is divided into three deep neural network training, respectively, calculated separately on each training wheels on deep modulation recognizer network training set and testing set of accuracy and losses, after the network convergence, to test the three networks in different SNR test data accuracies. 

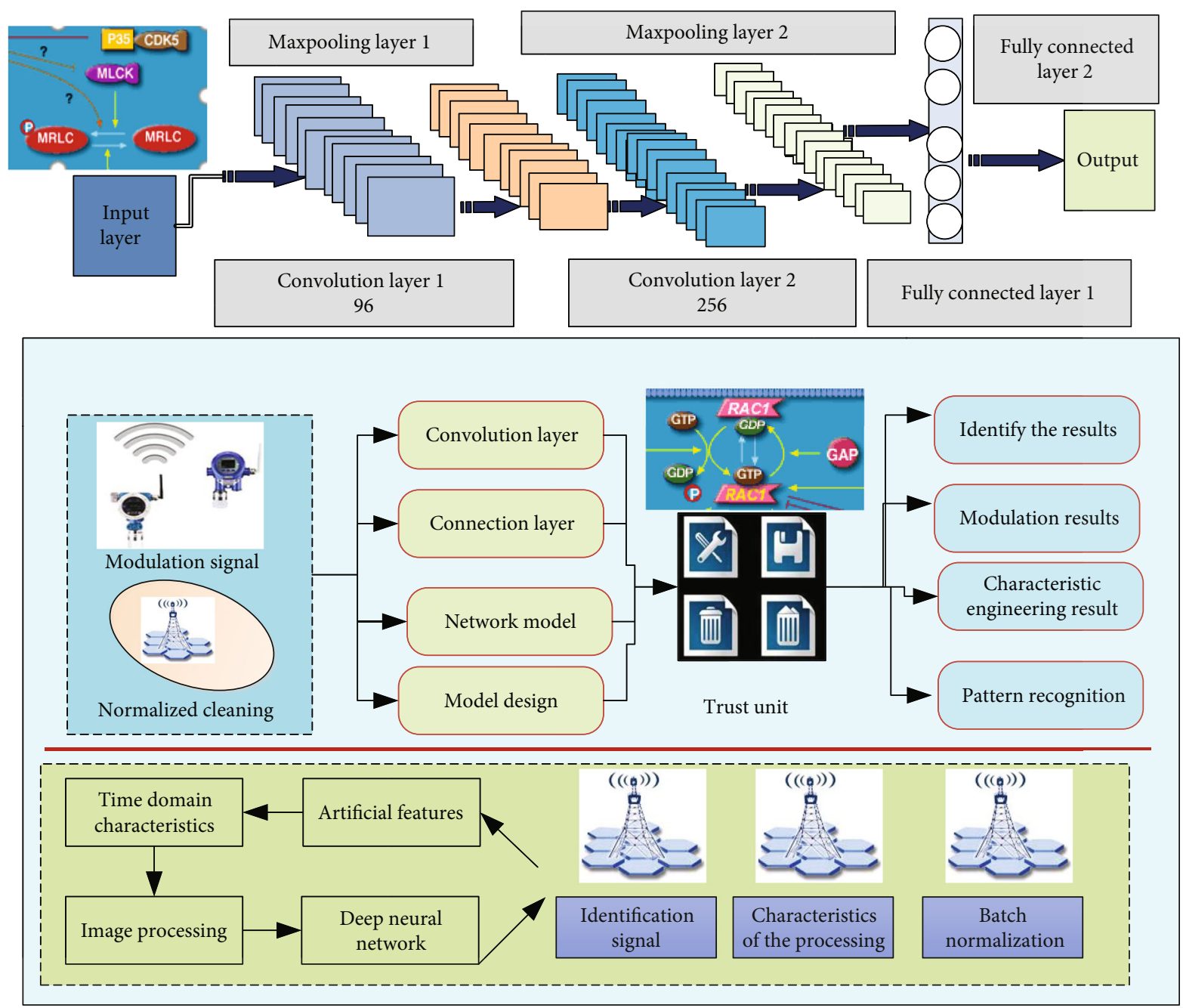

FIGURE 4: Flow chart of communication signal modulation pattern recognition based on deep neural network.

TABLE 2: Network super parameter settings.

\begin{tabular}{lc}
\hline Super parameter & Set the value \\
\hline Vector & 0.0001 \\
Attenuation coefficient of weight & 0.00006 \\
Number of iterations & 60 \\
Batch size & 129 \\
\hline
\end{tabular}

The number of neurons in the input layer and output layer of the neural network can be clearly obtained by considering the characteristic vector dimension of the signal and the modulation mode types of the signal to be recognized. The signal features we used in the analysis included 5 spectral features and 12 cumulant features, so each signal could be described by a 17-dimensional feature vector, so we set the number of neurons in the input layer as 17 . Since there are seven types of modulation signals we need to recognize, the number of neurons in the output layer is set as 7 . For the setting of the number of hidden layer neurons, we might as well consider setting a little larger. Because when the greater the number of hidden layer neurons, the greater the capacity of the neural network model, it is more easy to fitting the training data; that is to say, the performance of the neural network on the training set will be better, but it also means that the model had the greater risk than the fitting; we can have a very good avoidance over fitting strategy that is regularization. We can not only apply L2 regularization to the weight of the network but also randomly inactivate the neurons in the two hidden layers. Based on this, we can set the number of neurons in the hidden layer as 40 and 32 and set the random inactivation probability of neurons in the hidden layer as $P$ $=0.6$. According to the above description, the concrete structure of our modulation recognizer for deep neural network can be shown in Table 3 .

The first is the ReLU activation function, which is used for the activation of hidden layer neurons. ReLU activation function is not only simple to calculate but also can well alleviate the problem of gradient disappearance, which can greatly accelerate the convergence speed of neural network and improve the performance of neural network. Although the "dead zone" phenomenon exists in the ReLU function, it can be effectively alleviated by appropriate parameter initialization strategy. 


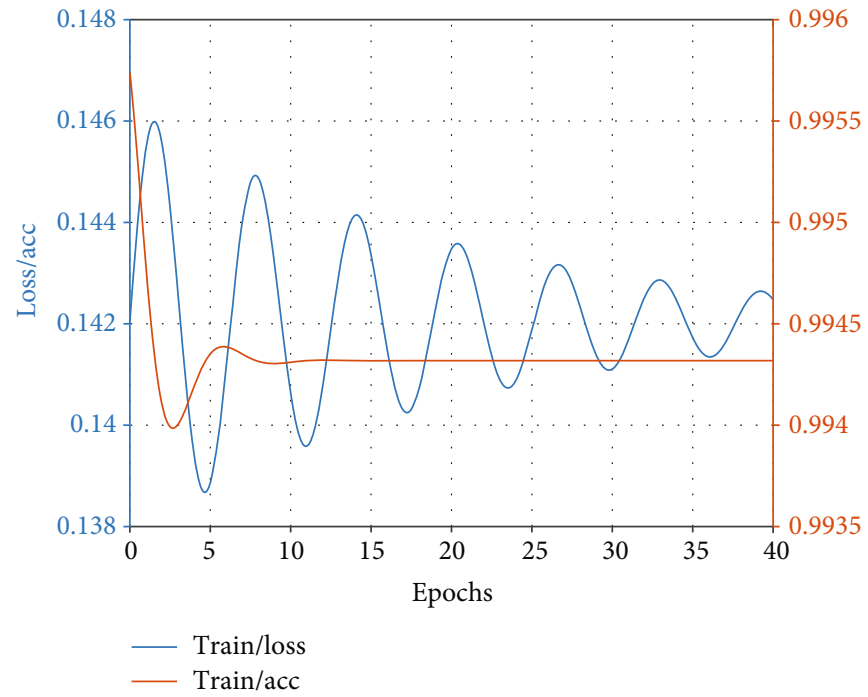

FIGURE 5: The relation between the loss value of validation set and the accuracy and the number of iterations.

TABLE 3: Specific structure of feedforward neural network.

\begin{tabular}{lccc}
\hline $\begin{array}{l}\text { Network } \\
\text { layer }\end{array}$ & $\begin{array}{c}\text { Input } \\
\text { dimensions }\end{array}$ & $\begin{array}{c}\text { Output } \\
\text { dimensions }\end{array}$ & $\begin{array}{c}\text { Number of } \\
\text { parameters }\end{array}$ \\
\hline $\begin{array}{l}\text { Densel } \\
\begin{array}{l}\text { Dropout } \\
(0.8)\end{array}\end{array}$ & (None, 18) & (None, 43) & 725 \\
$\begin{array}{l}\text { Den set } \\
\text { Dropout } \\
(0.7)\end{array}$ & $($ None, 41) & (None, 42) & 2 \\
Dense3 & $($ None, 31) & $($ None, 31) $)$ & 1332 \\
\hline & & $($ None, 6) $)$ & 1 \\
\end{tabular}

The second is the softmax activation function, which acts on the output nerve layer neurons. Softmax function can map the output to the probability of the communication signal corresponding to each modulation mode, so that the output of the neural network can form a probability distribution vector, which can be used to train the neural network modulation recognizer for the classification task with the crossentropy loss function.

As for the selection of the loss function, considering that the modulation pattern recognition of communication signals is essentially a multiclassification problem, the loss function can be explicitly designed as the cross-entropy loss function. Because the cross-entropy loss function is used as a measure of the difference between two probability distributions, the output layer using softmax activation function can ensure the output is a probability distribution vector dimensions for the output category number, so for real label samples, it must also be converted to a probability distribution of the same dimension vector to calculate cross office according to the loss. Hot codinq can achieve this goal. Therefore, we can consider the real taqs data of hot coding and convert it into multiple types of multidimensional vector modulation modes. In this hot vector alone, the modulated signal corresponds to the
TABLE 4: Signal modulation mode label and unique heat vector corresponding table.

\begin{tabular}{lc}
\hline Modulation mode label & Unique heat coding vector \\
\hline 0 & $(1,0,0,0,0,0,0)$ \\
1 & $(0,0,0,1,0,0,0)$ \\
2 & $(1,0,0,0,1,0,0)$ \\
3 & $(1,0,0,0,0,1,0)$ \\
4 & $(1,1,0,0,0,0,0)$ \\
5 & $(1,0,1,0,0,0,0)$ \\
6 & $(1,0,0,0,0,0,1)$ \\
\hline
\end{tabular}

model. One dimension of the corresponding vector is 1 , and the other dimensions are 0 . The specific corresponding relationship is shown in Table 4.

2.4. Collaborative Implementation of Deep Neural Network Communication Signal Modulation Identifier on CPU and GPU Platform. The first step is data preparation: in the deep neural network modulation identifier, its input is the feature vector extracted from the original communication signal sampling data, and the feature vector is preprocessed by the maximum and minimum normalization. The label calibration of the modulation method, the unique thermal vectorization of the label, and the division of the training set and the test set are analyzed.

The second step is to build the model: based on the Keras and TensorFlow libraries, this four-layer deep neural network is built on the computing platform of CPU and GPU according to the network structure described.

The third step is to train the model and test: the divided training dataset is used for training the neural network, and then, the test dataset is used to test the performance of the network. 


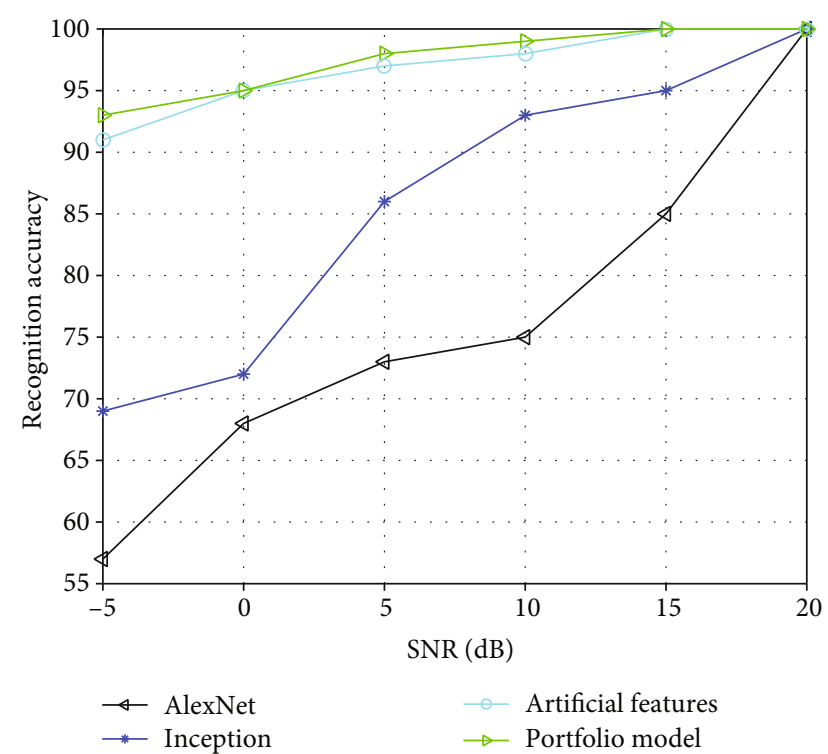

FIGURE 6: Recognition accuracy of different models for 18 kinds of simulation signals.

2.5. Experimental Verification. In the feature extraction part, a signal sampling sequence consisting of 40960 sampling points is intercepted. After feature extraction, 20-dimensional feature vectors can be obtained. Each simulation signal ranges from 1 $\mathrm{SdB}$ to $20 \mathrm{~dB}$. The features are extracted with different signalto-noise ratios. It can be found that comparing the two single model and modulation recognition model based on ergonomic 20-dimensional features, the combined model has achieved better results. At $-5 \mathrm{~dB}$, Inception-ResNet significantly improves the recognition effect of the V2 model by 93\% under the condition of low signal-to-noise ratio. And the recognition effect is slightly better than modulation recognition based on 20-dimensional human body characteristics. 1000 samples are generated every $2 \mathrm{~dB}$, with a ratio of $3: 1: 1$ By machine extraction, training set, verification set, and test set are added, respectively.

The combined model is based on the migrated InceptionResNet-V2 model. Figure 6 compares the recognition accuracy of the combined model with the three existing models in the previous two chapters for 18 kinds of simulation signals under different signal-to-noise ratios. Can be found, and the deep study of two single model and the modulation recognition model based on 20-D characteristics of human engineering, combined model has achieved better effect, when $-5 \mathrm{~dB}$ evidently improve recognition accuracy rate of 93\% combined the Inception-ResNet- before the V2 model recognition effect under the condition of low SNR, and the recognition effect is slightly better than for modulation recognition based on 20-D human characteristics.

It can be seen that the addition of 8 artificial feature parameters enables the combined model to provide a more effective feature expression for the signal, and it is effective to use the model to assemble the features online in the modulation identification. Combined model is compatible with the artificial features and advantages; based on timefrequency diagram of the automatic feature in the case of

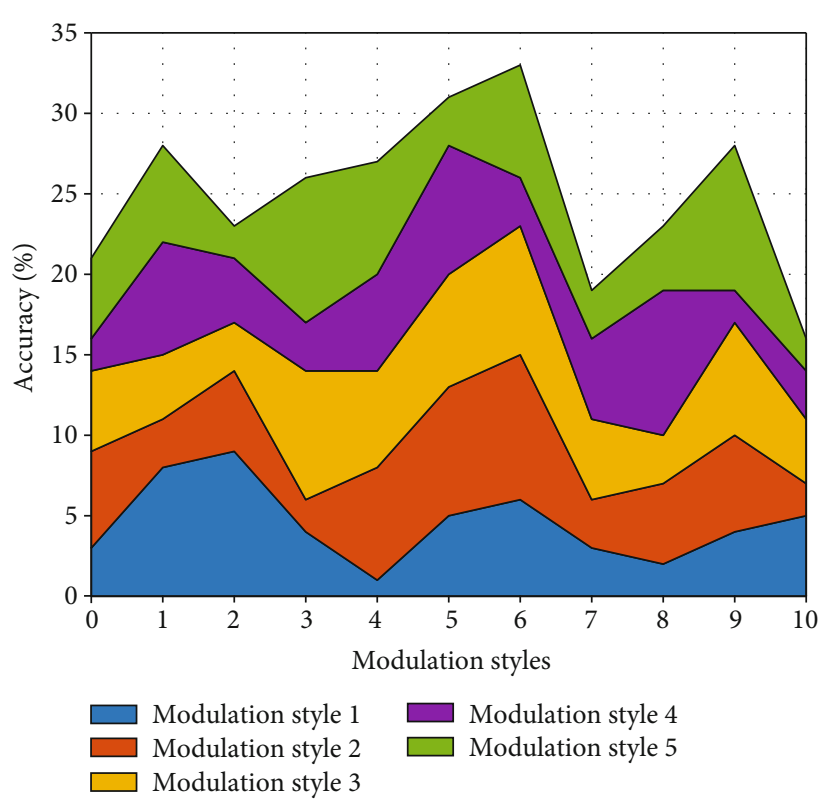

Figure 7: Confusion matrix of the combined model for 18 kinds of simulation signals at $11 \mathrm{~dB}$.

TABLE 5: Recognition rate of offline test.

\begin{tabular}{lcc}
\hline $\begin{array}{l}\text { Modulation } \\
\text { method }\end{array}$ & $\begin{array}{c}\text { Recognition rate } \\
(\mathrm{SNR}=16 \mathrm{~dB})\end{array}$ & $\begin{array}{c}\text { Recognition rate } \\
(\mathrm{SNR}=15 \mathrm{~dB})\end{array}$ \\
\hline 4ASK & $97.4 \%$ & $99.7 \%$ \\
2FSK & $94.3 \%$ & $98.3 \%$ \\
4FSK & $91.5 \%$ & $98.5 \%$ \\
8FSK & $89.1 \%$ & $93.2 \%$ \\
8PSK & $95.7 \%$ & $98.8 \%$ \\
8QAM & $92.2 \%$ & $96.3 \%$ \\
16QAM & $6.9 \%$ & $97.1 \%$ \\
32QAM & $94.5 \%$ & $98.2 \%$ \\
64QAM & $91.3 \%$ & $95.6 \%$ \\
AM & $91.6 \%$ & $95.3 \%$ \\
\hline
\end{tabular}

low signal-to-noise ratio (SNR), artificial features play an important role, and when the signal-to-noise ratio is not less than $7 \mathrm{~dB}$, using the migrated Inception-ResNet-V2 model can be the signal of time-frequency diagram that obtained the recognition accuracy higher than $95 \%$; accordingly, sectional model has achieved above $98 \%$ accuracy.

Figure 7 shows the confusion matrix of the combined model for 11 kinds of simulation signals at $11 \mathrm{~dB}$, where labels $0-10$ represent signals with different modulation styles. It can be seen that the combined model can realize the accurate recognition of the 11 simulation signals at $11 \mathrm{~dB}$.

The data of 4ASK, 2FSK, 4FSK, 8FSK, 8PSK, 8QAM, 16QAM, 32QAM, and 64QAM signals are generated by MATLAB. The generation method is as follows: 258 random baseband codes are generated, the codes are formed by the rising cosine filter, the roll drop coefficient is 0.6 , the corresponding modulation is carried out according to the selected modulation type, and the carrier frequency is $100 \mathrm{MHz}$. After 
TABLE 6: Test recognition rate of 16 kinds of communication signals.

\begin{tabular}{|c|c|c|c|}
\hline $\begin{array}{l}\text { Modulation } \\
\text { method }\end{array}$ & Test way & Signal source & $\begin{array}{l}\text { Recognition } \\
\text { rate }\end{array}$ \\
\hline $\mathrm{AM}$ & $\begin{array}{l}\text { Offline } \\
\text { test }\end{array}$ & $\begin{array}{c}\text { MATLAB to } \\
\text { produce }\end{array}$ & $95.3 \%$ \\
\hline FM & $\begin{array}{l}\text { Online } \\
\text { test }\end{array}$ & FM radio & $92.6 \%$ \\
\hline 1JSB & $\begin{array}{l}\text { Online } \\
\text { test }\end{array}$ & Signal generator & $98.6 \%$ \\
\hline LSB & $\begin{array}{l}\text { Online } \\
\text { test }\end{array}$ & Signal generator & $99.1 \%$ \\
\hline 2ASK & $\begin{array}{l}\text { Online } \\
\text { test }\end{array}$ & Signal generator & $96.7 \%$ \\
\hline 4ASK & $\begin{array}{l}\text { Offline } \\
\text { test }\end{array}$ & $\begin{array}{l}\text { MATLAB to } \\
\text { produce }\end{array}$ & $99.3 \%$ \\
\hline 2PSK & $\begin{array}{l}\text { Online } \\
\text { test }\end{array}$ & Signal generator & $98.4 \%$ \\
\hline 4PSK & $\begin{array}{l}\text { Online } \\
\text { test }\end{array}$ & Signal generator & $92.2 \%$ \\
\hline 8PSK & $\begin{array}{l}\text { Offline } \\
\text { test }\end{array}$ & $\begin{array}{l}\text { MATLAB to } \\
\text { produce }\end{array}$ & $98.5 \%$ \\
\hline 2FSK & $\begin{array}{l}\text { Offline } \\
\text { test }\end{array}$ & $\begin{array}{l}\text { MATLAB to } \\
\text { produce }\end{array}$ & $98.1 \%$ \\
\hline 4FSK & $\begin{array}{l}\text { Offline } \\
\text { test }\end{array}$ & $\begin{array}{l}\text { MATLAB to } \\
\text { produce }\end{array}$ & $98.4 \%$ \\
\hline 8FSK & $\begin{array}{l}\text { Offline } \\
\text { test }\end{array}$ & $\begin{array}{l}\text { MATLAB to } \\
\text { produce }\end{array}$ & $93.7 \%$ \\
\hline 8QAM & $\begin{array}{l}\text { Offline } \\
\text { test }\end{array}$ & $\begin{array}{l}\text { MATLAB to } \\
\text { produce }\end{array}$ & $96.3 \%$ \\
\hline 16QAM & $\begin{array}{l}\text { Offline } \\
\text { test }\end{array}$ & $\begin{array}{l}\text { MATLAB to } \\
\text { produce }\end{array}$ & $97.3 \%$ \\
\hline 32QAM & $\begin{array}{l}\text { Offline } \\
\text { test }\end{array}$ & $\begin{array}{l}\text { MATLAB to } \\
\text { produce }\end{array}$ & $98.2 \%$ \\
\hline 64QAM & $\begin{array}{l}\text { Offline } \\
\text { test }\end{array}$ & $\begin{array}{l}\text { MATLAB to } \\
\text { produce }\end{array}$ & $95.7 \%$ \\
\hline
\end{tabular}

AM signal is generated, a low-pass filter with a cut-off frequency of $8 \mathrm{kHz}$ is used for filtering. After filtering, 100 $\mathrm{MHz}$ carrier is used for modulation, and the depth of modulation is random value in the range of $[0.4,1]$. After modulation, noise is superimposed, and then, orthogonal down conversion and low-pass filtering are carried out. The filter bandwidth is $300 \mathrm{kHz}$, and the filtered IQ data is stored as a file.

The code that calls modulation recognition reads the IQ data file and performs modulation recognition, using 8195 data points for each identification. If it is identified as QAM signal, the entire IQ data file is read for identification. The recognition rate of each signal is shown in Table 5:

Test methods and test results of 16 signals are shown in Table 6 . For the modulation method that uses signal generator to generate signals for testing, the recognition rate is measured when the output power of signal source is $-82 \mathrm{dBm}$; for the modulation method that uses offline testing, the signalto-noise ratio of the recognition rate is $15 \mathrm{~dB}$.

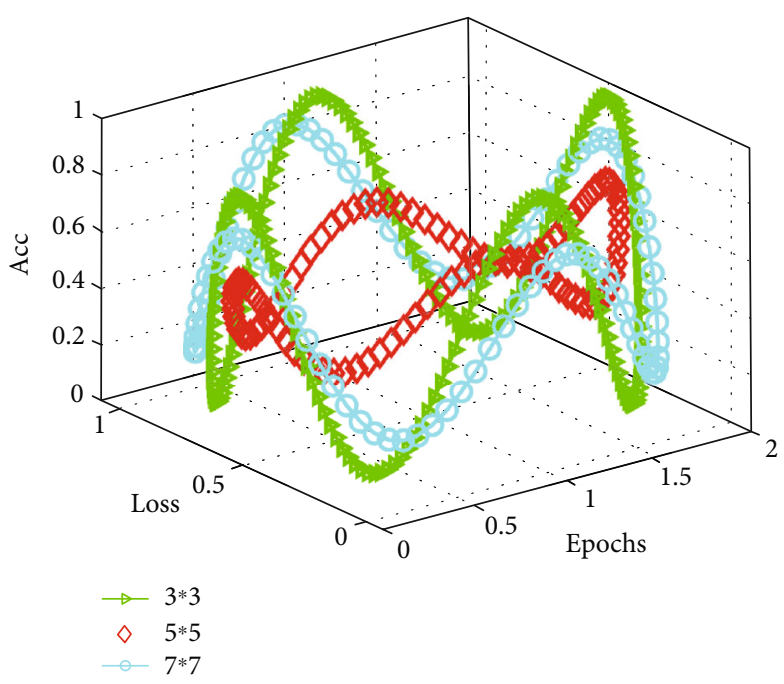

FIgURE 8: Relation between the accuracy of verification set and the number of training iterations for different deep cores.

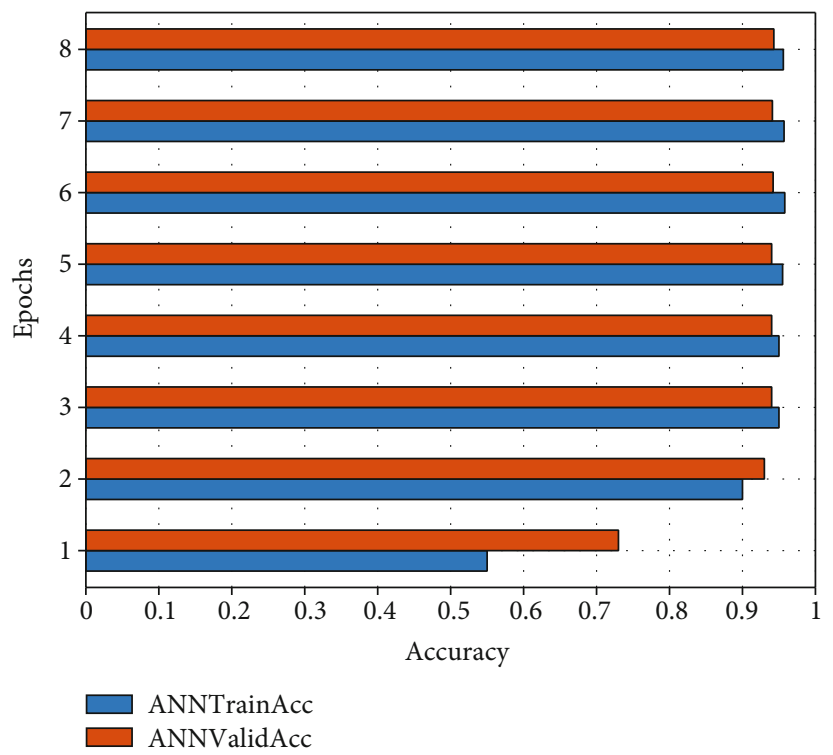

FIgURE 9: Accuracy performance curve of deep neural network on training set and verification set.

Deep nucleus size is usually odd, commonly $3 * 3,5 * 5$, etc., but there is no theoretical basis. The size of the deep core is usually determined by a large number of practical tests. And then there is a special deep core of $1 \times 1$, and deep cores of that size are usually used for dimension-reduction or linear transformations. The setting of the network hyperparameter is the same as that of the network hyperparameter in 4.3.2. After network training, network models with different deep core sizes were used to verify the accuracy of the set in the training process to analyze the influence of different deep core sizes on network performance. When the deep core size is different, the accuracy of the verification set changes with the increase of the number of training iterations, as shown in Figure 8: 
As can be seen from Figure 8, when the size of deep core is $3 * 3$, the accuracy of verification set is the highest, and with the increase of the number of training iterations, the fluctuation range of the accuracy of verification set is the smallest, and $3 * 3$ deep core will be used for deep calculation.

The training process of the deep neural network communication signal modulation recognitor is shown in Figure 9. The curve in Figure 9 represents the accuracy curve of the neural network on the training set and the verification set.

With the increase of the number of network training rounds, the accuracy of the deep neural network in the training set and the test set gradually improved and tended to be stable, and the modulation recognizer in the training set and the test set reached a high level of accuracy, both of which exceeded $98 \%$. This means that our neural network has been fully and effectively trained and no fitting phenomenon has occurred.

Through experimental verification, the online splicing features take into account the dual advantages of time-frequency analysis and artificial features, and the combined model design with better recognition effect is completed. Then, after selecting an appropriate feature extraction subnetwork, a triplet network model is built, and the similarity of the input timefrequency graph is calculated by using the Euclidean distance between the feature vectors, and a reasonable sample decision method is designed, which not only improves the matching degree between the input time-frequency graph samples and the Inception-ResNet-V2 model but also improves the matching degree between the input time-frequency graph samples and the Inception-ResNet-V2 model. It also realizes the recognition of the newly modulated signal and expands the application range of the existing model. Finally, the algorithm test and performance analysis based on the above two models are completed by using the real signal and simulation signal under different SNR.

\section{Conclusion}

Based on Keras and TensorFlow libraries, the deep neural network communication signal modulation recognizer is implemented on CPU and GPU hardware platform, and the training and testing tasks are completed. Experiments show that the deep neural network has good performance on both the training set and the test set, and can accurately identify the modulation mode of the communication signal with low SNR, which shows the robustness of the neural network. The deep neural network in this chapter had better reached the accuracy level of $97 \%$, and we can see that the modulation recognizer based on deep neural network can achieve a better accuracy level. Modulation recognizers for communication signals are constructed based on two kinds of mainstream deep neural network structures: three kinds of modulation recognizers are constructed based on deep neural network architecture, and their performance differences are compared; based on the cyclic neural network architecture, we construct two modulation identifiers and compare their performance differences. The experimental results show that the modulation recognizer based on deep neural network has a good accuracy performance on the sim- ulation dataset. For the modulation signal identification problem adopted in this paper, we try to collect more signal modulation modes to research more signal characteristics, so that the algorithm can identify more communication signal modulation modes.

\section{Data Availability}

Data sharing is not applicable to this article as no datasets were generated or analyzed during the current study.

\section{Consent}

Informed consent was obtained from all individual participants included in the study references.

\section{Conflicts of Interest}

We declare that there is no conflict of interest.

\section{Acknowledgments}

This study was supported by the National Natural Science Foundation of China: the research on ferromagnetic resonance temperature imaging based on superparamagnetic nanoparticles (Grant 61773018), research on low complexity coding model and method based on 3D-HEVC (Grant 61771432), research on information acquisition and processing method of temperature field in superparamagnetic nanoparticle targeted (Grant 61374014), and a real-time and accurate method for measuring $2 \mathrm{D}$ temperature distribution in magnetic nanoparticle-mediated hyperthermia (Grant 61803346).

\section{References}

[1] M. Richterova, "Signal modulation recognizer based on method of artificial neural networks," PIERS Online, vol. 1, no. 5, pp. 575-578, 2015.

[2] $\mathrm{S} . \mathrm{Wu}$, "Communication modulation recognition algorithm based on STFT mechanism in combination with unsupervised feature-learning network," Peer-to-Peer Networking and Applications, vol. 12, no. 3, pp. 1615-1623, 2019.

[3] Q. Guan and Y. Zhang, "Cyclic cumulant based on communication signal multilayer neural network modulation pattern recognition," IOP Conference Series Materials Science and Engineering, vol. 677, pp. 42113-42125, 2019.

[4] S. Peng, H. Jiang, and H. Wang, "Modulation classification based on signal constellation diagrams and deep learning," IEEE Transactions on Neural Networks and Learning Systems, vol. 6, no. 3, pp. 28506-28523, 2018.

[5] X. Bi, "A method for modulation recognition of maritime search and rescue communication signal based on neural network," Journal of Coastal Research, vol. 103, no. sp1, pp. 847862, 2020.

[6] P. Wu, B. Sun, and S. Su, "Automatic modulation classification based on deep learning for software-defined radio," Mathematical Problems in Engineering, vol. 2020, Article ID 2678310, 13 pages, 2020.

[7] Z. Y. Li and Y. D. Zhang, "DenseNet-ResNet-LSTM model for modulation recognition of communication signal," Journal of 
Physics: Conference Series, vol. 1693, no. 1, pp. 12150-12165, 2020.

[8] H. Hosseinzadeh, F. Razzazi, and A. Haghbin, "A self training approach to automatic modulation classification based on semi-supervised online passive aggressive algorithm," Wireless Personal Communications, vol. 82, no. 3, pp. 1303-1319, 2015.

[9] R. M. Al-Makhlasawy, A. A. Hefnawy, and E. MMA, "Modulation classification in the presence of adjacent channel interference using convolutional neural networks," International Journal of Communication Systems, vol. 33, no. 13, pp. 42954306, 2020.

[10] K. Li, W. Pan, and Y. Li, "A method to detect sleep apnea based on deep neural network and hidden Markov model using single-lead ECG signal," Neurocomputing, vol. 294, no. 7, pp. 94-101, 2018.

[11] L. Sun, J. Chen, and K. Xie, "Deep and shallow features fusion based on deep convolutional neural network for speech emotion recognition," International Journal of Speech Technology, vol. 21, no. 4, pp. 931-940, 2018.

[12] A. Lozano-Diez, R. Zazo, D. T. Toledano, and J. GonzalezRodriguez, "An analysis of the influence of deep neural network (DNN) topology in bottleneck feature based language recognition," PLoS ONE, vol. 12, no. 8, pp. e0182580e0182592, 2017.

[13] W. Shirui, Z. Yuelun, and L. Shubin, "Performance of deep neural network-based artificial intelligence method in diabetic retinopathy screening: a systematic review and meta-analysis of diagnostic test accuracy," European Journal of Endocrinology, vol. 2020, no. 183, pp. 41-49, 2020.

[14] Y. Yang, M. Chen, and Y. Wang, "Digital signal modulation classification using data conversion method based on convolutional neural network," Journal of Physics: Conference Series, vol. 1693, no. 1, pp. 12039-12050, 2020.

[15] R. Arunkumar and P. Karthigaikumar, "Multi-retinal disease classification by reduced deep learning features," Neural Computing \& Applications, vol. 28, no. 2, pp. 329-334, 2015.

[16] N. Teimouri, M. Omid, K. Mollazade, and A. Rajabipour, “An artificial neural network-based method to identify five classes of almond according to visual features," Journal of Food Process Engineering, vol. 39, no. 6, pp. 625-635, 2016.

[17] Y. Qian, N. Chen, and K. Yu, "Deep features for automatic spoofing detection," Speech Communication, vol. 85, pp. 43$52,2016$.

[18] J. Li and G. Jian, "A new feature extraction algorithm based on entropy cloud characteristics of communication signals," Mathematical Problems in Engineering, vol. 2015, Article ID 891731, 8 pages, 2015.

[19] Y. Yao, L. Yu, and Y. Chen, "Feature extraction method of radiation source in deep learning based on square integral bispectrum," Journal of Physics: Conference Series, vol. 1678, no. 1, pp. 12074-12080, 2020.

[20] Y. He, Y. Zhu, and W. Lin, "HTTP tunnel Trojan detection model based on deep learning," Journal of Physics: Conference Series, vol. 1187, no. 4, pp. 42055-42066, 2019.

[21] Y. Huang, W. Jin, and Z. Yu, "Radar emitter signal recognition based on deep learning and ensemble learning," Systems Engineering and Electronics, vol. 40, no. 11, pp. 2420-2425, 2018.

[22] M. Li, W. Chen, and Z. Tao, "A novel seizure diagnostic model based on kernel density estimation and least squares support vector machine," Biomedical Signal Processing and Control, vol. 41, no. 3, pp. 233-241, 2018.
[23] M. Sawalkar and V. V. Yerigeri, "Classification of fruit family based on features extraction using Penn classification," IOSR Journal of Electronics and Communication Engineering, vol. 13, no. 6, pp. 48-54, 2018.

[24] P. Heum, "Extraction of major features for major adverse cardiac events from KAMIR dataset based on deep learning algorithm," International Conference on Future Information \& Communication Engineering, vol. 9, no. 1, pp. 69-72, 2017.

[25] J. K. Kim, B. D. Kim, and D. W. Yoon, "Deep neural networkbased automatic modulation classification technique," The Journal of Korean Institute of Information Technology, vol. 14, no. 12, pp. 107-123, 2016.

[26] J. Han, N. Lin, J. Ruan, X. Wang, W. Wei, and H. Lu, “A model for joint planning of production and distribution of fresh produce in agricultural Internet of Things," IEEE Internet of Things Journal, 2020.

[27] W. Wang, N. Kumar, J. Chen et al., "Realizing the potential of the Internet of Things for smart tourism with 5G and AI," IEEE Network, vol. 34, no. 6, pp. 295-301, 2020.

[28] A. Zielonka, A. Sikora, M. Woźniak, W. Wei, Q. Ke, and Z. Bai, "Intelligent Internet of Things system for smart home optimal convection," IEEE Transactions on Industrial Informatics, vol. 17, no. 6, pp. 4308-4317, 2021.

[29] S. H. Ahmed, V. H. C. de Albuquerque, and W. Wei, "Guest editorial: special section on advanced deep learning algorithms for industrial Internet of Things," IEEE Transactions on Industrial Informatics, vol. 17, no. 4, pp. 2764-2766, 2021. 